\title{
Confounding in association studies: month of birth and multiple sclerosis
}

\author{
Barnaby Fiddes $\cdot$ James Wason $\cdot$ Stephen Sawcer
}

Received: 11 December 2013/ Accepted: 2 January 2014/Published online: 12 January 2014

(C) The Author(s) 2014. This article is published with open access at Springerlink.com

\begin{abstract}
Association studies form the backbone of biomedical research, with almost every effort in the field ultimately boiling down to a comparison between groups, coupled with some form of statistical test intended to determine whether or not any observed difference is more or less than would be expected by chance. Unfortunately, although the paradigm is powerful and frequently effective, it is often forgotten that false positive association can easily arise if there is any bias or systematic difference in the way in which study subjects are selected into the considered groups. To protect against such confounding, researchers generally try to match cases and controls for extraneous variables thought to correlate with the exposures of interest. However, if seemingly homogenously distributed exposures are actually more heterogeneous than appreciated, then matching may be inadequate and false positive results can still arise. In this review, we will illustrate these fundamental issues by considering the previously proposed relationship between month of birth and multiple sclerosis. This much discussed but false positive association serves as a reminder of just how heterogeneous even easily measured environmental risk factors can be, and how easily case
\end{abstract}

Electronic supplementary material The online version of this article (doi:10.1007/s00415-014-7241-y) contains supplementary material, which is available to authorized users.

B. Fiddes · S. Sawcer $(\bowtie)$

Department of Clinical Neurosciences, University of Cambridge, Cambridge Biomedical Campus, Hills Road,

Cambridge CB2 0QQ, UK

e-mail: sjs1016@mole.bio.cam.ac.uk; sjs1016@cam.ac.uk

J. Wason

Medical Research Council Biostatistics Unit,

Cambridge CB2 OSR, UK control studies can be confounded by seemingly minor differences in ascertainment.

Keywords Confounding - Association - Multiple sclerosis · Month of birth

\section{Introduction}

Establishing what's different about those who develop a disease as compared to those who remain unaffected (epidemiology) seems like it should be a fairly straightforward way of identifying clues to aetiology. In practice, however, such studies are surprisingly vulnerable to subtle biases that can easily generate false positive associations $[4,13]$. Even when an exposure can be accurately measured and does not change over time, such as an individual's genotype [or month of birth (MOB)], it is still possible for differences in ascertainment to result in apparently significant differences between cases and controls in the absence of any real effect, if the frequency of the exposure differs between sub-groups of the population considered [5, 31]. In genome-wide association screens, the large number of variants studied enables investigators to quantify and compensate for the influence of potentially confounding factors such as ancestry [17]. On the other hand, in studies considering individual risk factors (genetic or environmental), researchers cannot undertake such correction and instead are often forced to make simplifying assumptions, such as that within a given country exposure is likely to be uniform, and therefore that any confounding arising because of differences in how cases and controls are ascertained across the country is unlikely to be significant. For many risk factors this assumption is safe; for example, it certainly seems to be true for the vast majority of 
common genetic variants [3]. However, in the context of environmental risk factor analysis, the assumption of homogeneity of exposure has rarely been tested.

\section{Heterogeneity in the timing of birth}

Although there are many local and personal factors that might influence an individual's MOB, it seems reasonable to imagine that across the population in any given country these effects would likely average out; indeed, intuitively it feels unlikely that the probability of being born in any given month would vary between different parts of the same country, and equally unlikely that this probability might be significantly different in different years. In this context, it is unsurprising that the studies that have looked for association between multiple sclerosis and MOB have all assumed some degree of such homogeneity $[2,8,11,12$, $16,23-25,27-29,32,33]$. Unfortunately, it turns out that this assumption is invalid, and that confounding rather than biology has likely generated the apparent associations that have previously been reported [9]. The fact that MOB is extremely heterogeneous in the general population is well known in the anthropology literature [7, 15, 19-22], but seems to have gone largely un-noticed by those studying MOB as a potential risk factor in multiple sclerosis.
If the underlying birth rate in a country remained constant over time, we would only expect random fluctuations in the ratio between the observed and the expected number of births seen in any given month; with the $95 \%$ confidence interval on this ratio being $0.97-1.03$ in a country like Norway (population circa 5 million) and $0.99-1.01$ in a country like the UK (population circa 60 million). Surprisingly, this ratio shows much greater variation. Figure 1 shows the actual ratio of observed to expected births in each month present in 824 year and country-specific MOB records obtained from the national statistics available online from 16 European countries (as we described previously [9] ). Each of these 824 records is statistically significantly different from that expected assuming a constant birth rate, with all but three records remaining significant even after stringent Bonferroni correction (i.e. having $p<6 \times 10^{-5}$ ). Even when comparing each record with the number of expected births calculated by averaging across all the records for the corresponding country (ignoring 1/29 of the February births in leap years so that all records considered are based on 365 days per year), 807/824 records are significantly different (735/824 after Bonferroni correction). Furthermore, all 824 of the records include at least one spring month (March, April, May) where there is an excess in the birth rate and/or at least one winter month (November, December or January) showing a deficit. In more than

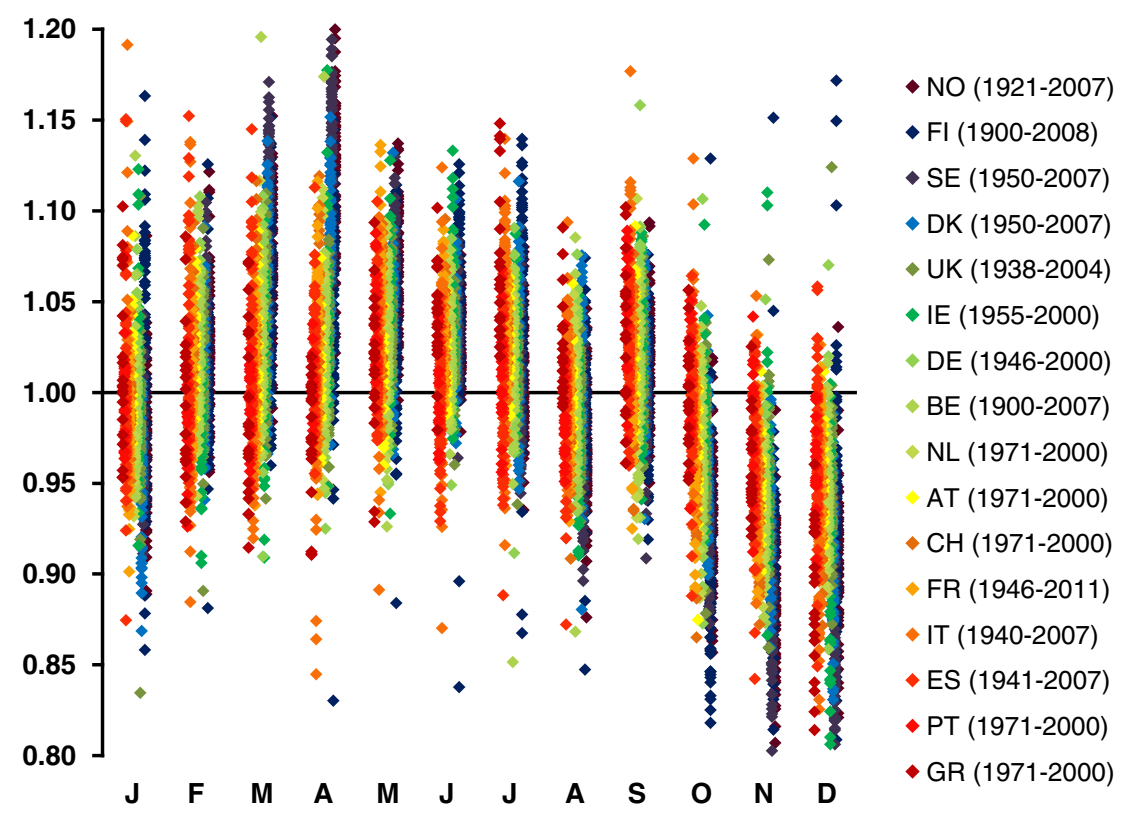

Fig. 1 Year and country-specific month of birth records from 16 European countries (Greece, Portugal, Spain, Italy, France, Switzerland, Austria, Netherlands, Belgium, Germany, Ireland, UK, Denmark, Sweden, Finland and Norway) [9]. The $x$ axis shows the months of the year (coded by their first letter), while the $y$ axis shows the ratio between the observed number of births in a month and the number of births that would have been expected if birth rate had remained constant throughout the corresponding year. Ratios were calculated allowing for the length of each month and for leap years, but for simplicity are plotted assuming the length of each month is equal. For clarity, the country specific records are offset on the $x$-axis according to latitude and heat coded. The legend indicates the range of years included and the two letter country code. A total of 824 year and country-specific records are shown (including over 270 million births) 


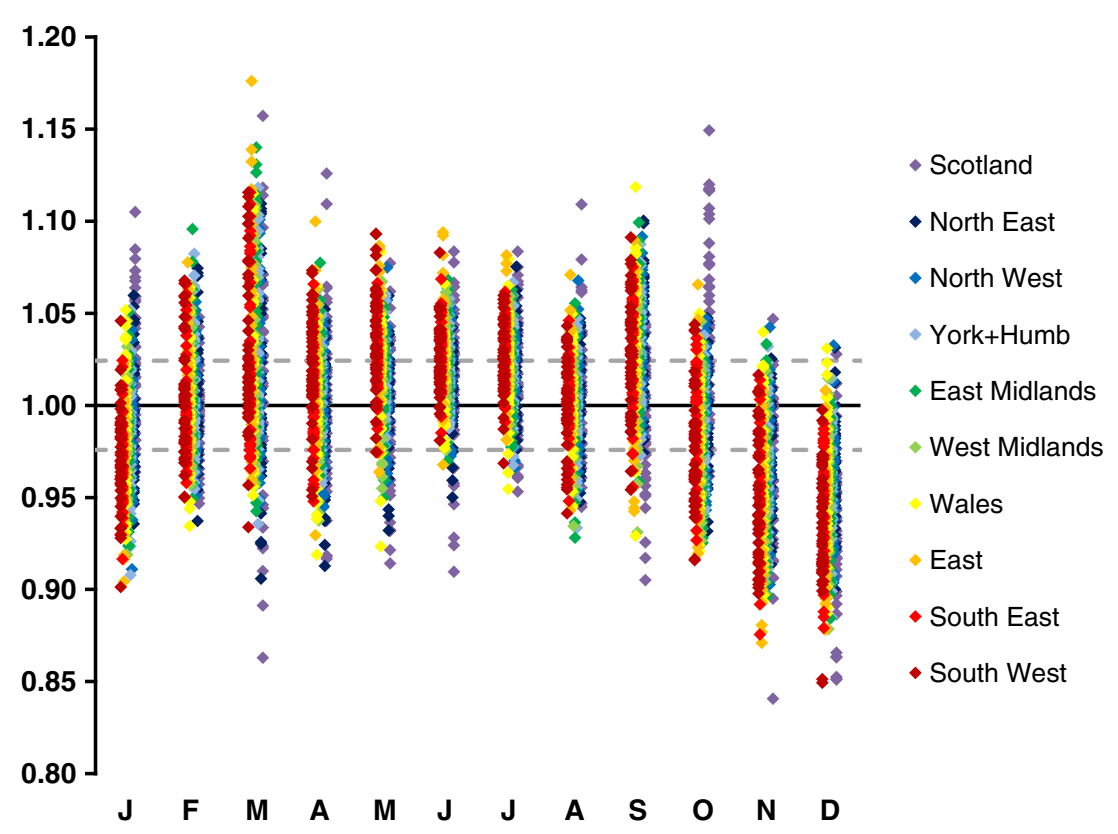

Fig. 2 MOB records for individual UK Government Office Regions (GOR) over the period 1965-2008. For administrative purposes, the UK is currently divided into 11 regions (each with a roughly equivalent population, circa 6 million); however, in the past, London was included as part of the South East and only ten regions were considered [9]. Since some records predate this spilt, we have considered London and the South East together in all records, and only considered the ten GOR. Again for clarity, individual regions are

$70 \%(596 / 824)$ of the records, at least one of these differences is statistically significant. Some $88 \%$ of records are significantly different from the preceding year in the same country. Figure 2 shows that even within a country there is marked and highly significant heterogeneity in birth rate, with $99 \%$ of records (437/440) for individual UK Government Office Regions being significantly different from that expected if birth rate were constant and $85 \%$ of records (374/440) are significantly different from the average across all records from the corresponding region. These data confirm that birth rate is subject to marked seasonal variation and allows us to unequivocally reject the assumption that birth rate is homogeneous.

\section{Heterogeneity in the distribution of multiple sclerosis}

The frequency of multiple sclerosis also shows considerable variation both between $[14,26]$ and within countries $[1,30,34]$. Because of this variation, even comprehensive case collections that have been established through national registries in a single country will inevitably include a disproportionate number of individuals from some regions (those with higher prevalence) and an under-representation of those from other regions (those with lower prevalence). Furthermore, because the incidence of multiple sclerosis is heat coded and slightly offset on the $x$-axis. Nota bene until very recently Scottish statistics were based on the month of birth registration rather than the actual month of birth. A total of 440 year and GOR-specific records are shown (including over 32 million births, data obtained from UK National Statistics Office-www. statistics.gov.uk). The dotted lines indicate the $95 \%$ confidence interval that would be expected for these regions (based on their population size) if the underlying birth rate were constant

age-dependent [6], any set of prevalent cases will necessarily be heterogeneous with respect to year of birth; including an excess of middle-aged individuals (the peak risk group) and smaller numbers of very young or much older individuals. The sort of variation in regional origin and year of birth that would be expected within a set of cases from a given country is illustrated in the Supplementary Figure S1. Cohorts collected through the efforts of interested researchers are likely to be even less representative of the country as a whole, as such collections are invariably biased in favour of prevalent cases from regions local to the interested investigator(s).

\section{Mismatching of cases and controls}

Given that seasonality of birth and multiple sclerosis are both highly heterogeneous with respect to geography and time, any mismatching for these extraneous variables between cases and controls has the potential to generate a spurious difference in the MOB pattern between these groups; this apparent association only reflecting differences in the regional and temporal origin of the two groups, rather than any genuinely causal effect. While we would expect such differences to get smaller as sample size increases, they will not tend toward zero unless cases and 


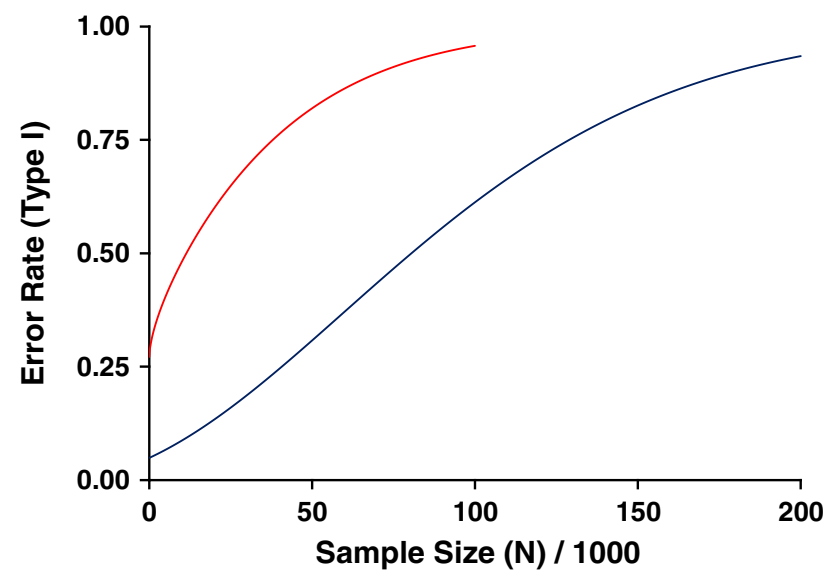

Fig. 3 Conservative estimate for the type I (false positive) error rate expected in MOB studies of differing size $(N=$ the number of cases $=$ the number of controls, in thousands), reproduced with permission from our original publication [9]. The lower curve indicates the probability of identifying any month showing a significant difference; $p$ value $<0.0042(=0.05$ Bonferroni corrected for the number of months), while the upper curve indicates the probability of seeing a nominally significant excess in at least one spring month and/or a nominally significant deficit in at least one winter month

controls are fully matched for regional origin and year of birth. Since the significance of any given difference tends to increase as sample size increases, the likelihood of seeing a significant false positive association as a result of mismatching actually increases as sample size increases [18]. Thus, although calculating the number of births expected in each month by averaging over available national birth statistics is mathematically easy and seems intuitively reasonable, the resulting estimates are only appropriate if the case collection tested has the same regional and temporal distribution. Unfortunately, none of the studies that have assessed MOB as a risk factor for multiple sclerosis have adequately matched their cases and controls for both regional origin and year of birth, making it highly likely that the reported associations are false positives. Figure 3 shows a conservative estimate for the rate of false positive association expected, assuming that controls are based on averaged national statistics, while case recruitment is weighted by the typical prevalence and year of birth data shown in supplementary figure $\mathrm{S} 1$ and as shown previously [9].

\section{Why do the results from MOB studies in multiple sclerosis seem to be consistent?}

Although it remains unclear exactly what factors drive the extensive variation in MOB that is apparent in national birth statistics, it is well established that there is a highly significant correlation between latitude and birth rate that is positive in spring months (March, April, May) and negative in winter months (November, December or January) [19, 21, 22]. Furthermore, there is significant evidence that these correlations have declined over time, such that there is much less seasonality in birth in today's developed world than was apparent previously $[7,15,20,21]$. It has been suggested that photoperiod (the hours of daylight in a day) might be responsible for the correlation with latitude, and it has also been suggested that perhaps the decline in these latitudinal gradients over time reflects our increasing ability to control our environment (through lighting and heating), and thus disconnect ourselves from the influence of such seasonal variables [10]. While these latitudinal and temporal correlations only account for a small fraction of the observed variation in birth rate, they do mean that there is an inevitable tendency for cases (which are generally older and more northern than the full set of individuals included in population-based birth statistics) to show higher rates of birth in spring months and lower rates of birth in winter months [9]. Coupled with the latitudinal gradient in the frequency of multiple sclerosis, these trends explain the rather superficial consistency in the MOB pattern considered typical of multiple sclerosis. These correlations favour the emergence of an apparent increase in risk during spring and reduction in risk during winter (see Fig. 4). Figure 3 shows a conservative estimate for the false positive replication of the MOB pattern considered typical of multiple sclerosis; i.e. a nominally significant excess in at least one spring month (March, April, May), and/or a nominally significant reduction in at least one winter month (November, December or January). These false positive rates are conservative, as they ignore the inevitable heterogeneity in MOB present within each of the individual Government Office Regions.

\section{What level of matching is required?}

As the population in a country is divided into smaller and smaller regional groups, the variance in the ratio of observed to expected births per month that results from random sampling will increase. At some point, this sampling variance will overwhelm the systematic effects driving the variation evident at the whole country level, and no heterogeneity in MOB will be apparent within such groups. Based on the variance in the ratio of observed to expected births per month evident at the whole country level (Fig. 1), we would anticipate that there would be little or no power to demonstrate heterogeneity in $\mathrm{MOB}$ in populations where the average total birth rate is $<1,200$ per year. Supplementary Figure S2 shows the MOB data from the 9-year period 2000-2008 for the 195 Local Authority regions from England and Wales that had an 


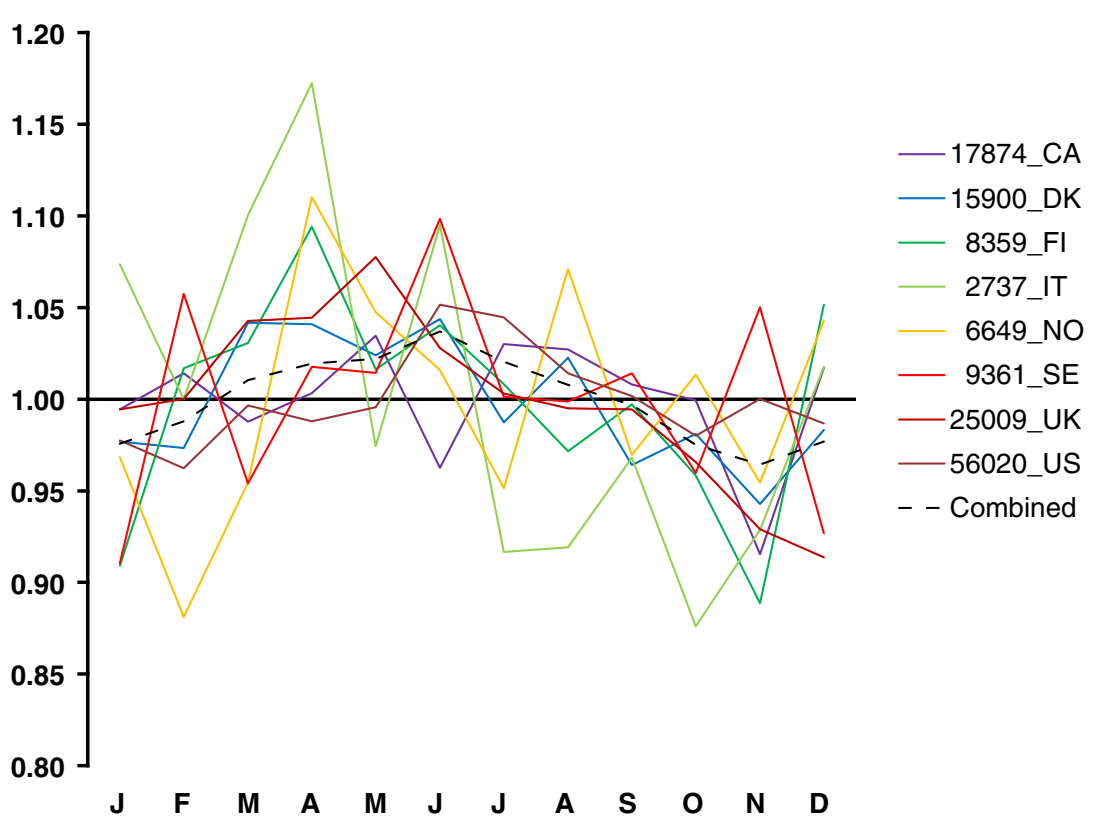

Fig. 4 Country specific multiple sclerosis MOB data from previously published reports $[2,8,9,12,16,23,25,29,32,33]$. The $x$-axis indicates the month of the year (as in Fig. 1), while the $y$ axis indicates the ratio of observed to expected birth in each month as previously reported, (see individual publications for the details of which national statistics were used to calculate the expected

average annual birth rate of more than 1,200 over that time period. Since each of these local authorities has a population of only approximately 200,000, the range of ratio values is much greater than in the GOR (population circa 6 million, Fig. 2), while the power to demonstrate these differences as significant is limited. In fact, $15 \%$ of these records show statistically significant evidence for seasonality. Given the decline in seasonality known to have occurred over the last century, it seems highly likely that local authority data from previous decades would be even more highly structured. These data confirm that MOB is heterogeneous down to the Local Authority level, and suggest that population statistics of corresponding resolution would likely be necessary to adequately control for year of birth and regional origin in an analysis of MOB as a risk factor in multiple sclerosis. It is unlikely that such detailed data exist in most countries. The extensive range of possible values for the ratio of births evident in the Supplementary Figure S2 also explains why studies considering cases collected in a single centre are likely to identify effects that are apparently larger than those seen in studies considering nationally recruited cases [2]. The greater variance in the smaller denominator population from which the cases are drawn necessarily exceeded anything seen as a result of systematic effects.

Using unaffected siblings as a source of controls is a logical way to try and reduce the confounding due to numbers). The legend indicates the number of cases studied together with the standard two letter country code. The dotted line indicates the ratio based on combining all the available data; the tendency to excess in spring and deficit in winter is apparent, as is the extreme heterogeneity between the studies

differences in regional origin [12, 27, 33]; however, these special individuals are again drawn from a much smaller denominator population, and of course are necessarily unmatched for year of birth. In multiple sclerosis, results using such controls have been inconsistent with each other and are too few in number to enable any confident assessment.

\section{Conclusion}

Although intuitively it seems reasonable to conclude that comparing the MOB seen in a group of cases with that expected based on averaged national birth statistics from the same country should provide a robust way of assessing the role of MOB as a risk factor, in reality, the extensive and highly significant variation in MOB that is present in the general population means that the inevitable heterogeneity in cases with respect to regional origin or year of birth frequently generates false positive association [9]. Unfortunately, the high rate of false positive association likely to arise as result of this under-recognised structure means that it is very likely that previous reports of association are false positive, and that in fact there is no MOB association in multiple sclerosis. These observations underline how easily false positive associations can arise when a tested exposure is wrongly assumed to be homogeneous. Many environmental factors are highly heterogeneous within the general 
population (e.g. smoking and vitamin D levels), raising the possibility that hidden structure could also undermine the testing of these variables. These observations serve to remind us that controlling for confounding needs to be as comprehensive in the analysis of candidate environmental risk factors as it is in genetics; exactly how this could be done is not immediately clear.

Acknowledgments This work was supported by the Cambridge NIHR Biomedical Research Centre. JW is supported by the Medical Research Council (G0800860) and BF is supported by a Raymond and Beverley Sackler studentship.

\section{Conflicts of interest None.}

Open Access This article is distributed under the terms of the Creative Commons Attribution License which permits any use, distribution, and reproduction in any medium, provided the original author(s) and the source are credited.

\section{References}

1. Ahlgren C, Oden A, Lycke J (2011) High nationwide prevalence of multiple sclerosis in Sweden. Mult Scler 17:901-908

2. Bayes HK, Weir CJ, O'Leary C (2010) Timing of birth and risk of multiple sclerosis in the Scottish population. Eur Neurol 63:36-40

3. Burton PP, Clayton D, Cardon L, Craddock N, Deloukas P, Duncanson A, Kwiatkowski DP, McCarthy MI (2007) Genomewide association study of 14,000 cases of seven common diseases and 3,000 shared controls. Nature 447:661-678

4. Cardon LR, Bell JI (2001) Association study designs for complex diseases. Nat Rev Genet 2:91-99

5. Cardon LR, Palmer LJ (2003) Population stratification and spurious allelic association. Lancet 361:598-604

6. Compston A, Confavreux C, Lassmann H, McDonald I, Miller D, Noseworthy J, Smith K, Wekerle H (2006) McAlpine's multiple sclerosis. Churchill Livingstone, London

7. Cummings DR (2010) Seasonal sunshine and vitamin D: a possible explanation for differences in European and United States birth patterns. Biodemography Soc Biol 56:105-122

8. Dobson R, Giovannoni G, Ramagopalan S (2013) The month of birth effect in multiple sclerosis: systematic review, meta-analysis and effect of latitude. J Neurol Neurosurg Psychiatry 84:427-432

9. Fiddes B, Wason J, Kemppinen A, Ban M, Compston A, Sawcer S (2013) Confounding underlies the apparent month of birth effect in multiple sclerosis. Ann Neurol 73:714-720

10. Foster RG, Roenneberg T (2008) Human responses to the geophysical daily, annual and lunar cycles. Curr Biol 18:R784-R794

11. Givon U, Zeilig G, Dolev M, Achiron A (2012) The month of birth and the incidence of multiple sclerosis in the Israeli population. Neuroepidemiology 38:64-68

12. Grytten N, Torkildsen O, Aarseth JH, Benjaminsen E, Celius EG, Dahl OP, Holmoy T, Loken-Amsrud K, Midgard R, Myhr KM, Risberg G, Vatne A, Kampman MT (2013) Month of birth as a latitude-dependent risk factor for multiple sclerosis in Norway. Mult Scler 19:1028-1034

13. Ioannidis JP (2005) Why most published research findings are false. PLoS Med 2:e124

14. Koch-Henriksen N, Sorensen PS (2010) The changing demographic pattern of multiple sclerosis epidemiology. Lancet Neurol 9:520-532
15. Lerchl A, Simoni M, Nieschlag E (1993) Changes in seasonality of birth rates in Germany from 1951 to 1990. Naturwissenschaften 80:516-518

16. Menni C, Lowell WE, Bentzen J, Bergamaschi R, Martinelli Boneschi F, Martinelli V, Bernardinelli L, Stenager E, Davis GE Jr, Foco L (2012) Short and long term variation in ultraviolet radiation and multiple sclerosis. Int J Environ Res Public Health 9:685-697

17. Price AL, Patterson NJ, Plenge RM, Weinblatt ME, Shadick NA, Reich D (2006) Principal components analysis corrects for stratification in genome-wide association studies. Nat Genet 38:904-909

18. Pritchard JK, Rosenberg NA (1999) Use of unlinked genetic markers to detect population stratification in association studies. Am J Hum Genet 65:220-228

19. Randall W (1993) Photoperiod, temperature, and regional patterns of conceptions in the USA. Int J Biometeorol 37:52-60

20. Randall W, Moos WS (1993) The 11-year cycle in human births. Int J Biometeorol 37:72-77

21. Roenneberg T (2004) The decline in human seasonality. J Biol Rhythms 19:193-195 discussion 196-197

22. Roenneberg T, Aschoff J (1990) Annual rhythm of human reproduction: I. Biology, sociology, or both? J Biol Rhythms 5:195-216

23. Saastamoinen KP, Auvinen MK, Tienari PJ (2012) Month of birth is associated with multiple sclerosis but not with HLADR15 in Finland. Mult Scler 18:563-568

24. Salemi G, Ragonese P, Aridon P, Reggio A, Nicoletti A, Buffa D, Conte S, Savettieri G (2000) Is season of birth associated with multiple sclerosis? Acta Neurol Scand 101:381-383

25. Salzer J, Svenningsson A, Sundstrom P (2010) Season of birth and multiple sclerosis in Sweden. Acta Neurol Scand 121:20-23

26. Simpson S Jr, Blizzard L, Otahal P, Van der Mei I, Taylor B (2011) Latitude is significantly associated with the prevalence of multiple sclerosis: a meta-analysis. J Neurol Neurosurg Psychiatry $82: 1132-1141$

27. Sotgiu S, Pugliatti M, Sotgiu MA, Fois ML, Arru G, Sanna A, Rosati G (2006) Seasonal fluctuation of multiple sclerosis births in Sardinia. J Neurol 253:38-44

28. Staples J, Ponsonby AL, Lim L (2010) Low maternal exposure to ultraviolet radiation in pregnancy, month of birth, and risk of multiple sclerosis in offspring: longitudinal analysis. BMJ 340: c1640

29. Templer DI, Trent NH, Spencer DA, Trent A, Corgiat MD, Mortensen PB, Gorton M (1992) Season of birth in multiple sclerosis. Acta Neurol Scand 85:107-109

30. Visser EM, Wilde K, Wilson JF, Yong KK, Counsell CE (2012) A new prevalence study of multiple sclerosis in Orkney, Shetland and Aberdeen city. J Neurol Neurosurg Psychiatry 83:719-724

31. Wacholder S, Rothman N, Caporaso N (2000) Population stratification in epidemiologic studies of common genetic variants and cancer: quantification of bias. J Natl Cancer Inst 92:11511158

32. Wiberg MA, Templer DI (1994) Season of birth in multiple sclerosis in Sweden: replication of Denmark Findings. J Orthomol Med 9:71-74

33. Willer CJ, Dyment DA, Sadovnick AD, Rothwell PM, Murray TJ, Ebers GC (2005) Timing of birth and risk of multiple sclerosis: population based study. BMJ 330:120

34. Zajicek JP, Ingram WM, Vickery J, Creanor S, Wright DE, Hobart JC (2010) Patient-orientated longitudinal study of multiple sclerosis in south west England (The South West Impact of Multiple Sclerosis Project, SWIMS) 1: protocol and baseline characteristics of cohort. BMC Neurol 10:88 